\title{
Influence of Magnetism on Second Harmonic Generation
}

\author{
J. Reif, ${ }^{1, *}$ C. Rau, ${ }^{2}$ and E. Matthias ${ }^{1}$ \\ ${ }^{1}$ Fachbereich Physik, Freie Universität Berlin, Arnimallee 14, 14195 Berlin, Germany \\ ${ }^{2}$ Physics Department, Rice University, P.O. Box 1892, Houston, Texas 77251
}

(Received 30 April 1993)

\begin{abstract}
Optical second harmonic generation (SHG) in $75^{\circ}$ reflection was studied on longitudinally magnetized $\mathrm{PtMnSb}(111)$ and magnetic circular dichroism was observed with SHG. For circularly polarized incident light of $532 \mathrm{~nm}$ it amounts to $(7 \pm 1) \%$. For linearly polarized incident radiation this leads to a nonlinear magneto-optical Kerr rotation of $(14 \pm 2)^{\circ}$. The strong symmetry-breaking effect of the magnetization is demonstrated by a $90^{\circ}$ turn of the $\mathrm{SH}$ polarization ellipse with regard to the linear polarization of the fundamental light.
\end{abstract}

PACS numbers: $78.20 . \mathrm{Ls}, 42.65 . \mathrm{Ky}$

Stimulated by theoretical and experimental progress as well as technological demands, magnetism of surfaces, alloys, thin films, and multilayer systems has recently received increased attention. Of primary interest are anisotropic effects which result from the distortion of the electronic charge distribution induced by the spin-orbit interaction. Examples are the existence of an electric field gradient in cubic crystals [1], anisotropic electric conductivity [2], magnetic dichroism in $\mathrm{x}$-ray adsorption [3-6] and photoemission [7,8], and the Faraday and the magneto-optical Kerr effect (MOKE) [9,10]. In this context it has been pointed out $[11,12]$ and experimentally demonstrated $[13,14]$ that optical second harmonic generation ( $\mathrm{SHG}$ ) is a technique particularly well suited for monitoring the symmetry reduction caused by magnetization. Although this technique is experimentally easy to handle under both normal and UHV conditions, it has up to now not received the attention that rhymes with its potential. The theory for the nonlinear magneto-optical Kerr effect (NOLIMOKE) has been worked out by Hübner and Bennemann [12], who predicted the spectral dependence of the nonlinear susceptibility for nickel. Aktsipetrov, Braginskii, and Esikov [13] studied SHG both in transmission and reflection on magnetized epitaxial $\mathrm{Y}_{2.5} \mathrm{Bi}_{0.5} \mathrm{Fe}_{5} \mathrm{O}_{12}$ films on an $\mathrm{Y}_{3} \mathrm{Fe}_{5} \mathrm{O}_{12}$ substrate. They reported a magneto-optical Kerr rotation between $1^{\circ}$ and $4^{\circ}$, dependent on film thickness but nevertheless significantly larger compared to the linear Kerr rotation. Reif et al. [14] showed that SHG is a sensitive technique for detecting surface magnetism of ferromagnetic crystals in UHV. We are not aware of any reports on magnetic circular dichroism in SHG, although in analogy to magnetic circular x-ray dichroism [3-6], this quantity is expected to yield information about the occupation of spinup and spin-down states in the conduction band [12-15].

It is the purpose of this Letter to demonstrate the influence of magnetization on SHG. The observed large effects on SHG in reflection are caused by two effects. First, with magnetic ordering the symmetry of the nonlinear susceptibility tensor $\chi^{(2)}$ is changed, compared to the nonmagnetic situation $[11,13,16]$. Second, the strength of the spin-orbit interaction determines the size of the tensor components [12]. It also introduces the sensitivity to the magnetization direction with regard to the $k$ vector of the incident light. To prove these points, we present the first data on magnetic circular dichroism in SHG, which amounts to an asymmetry of $(7 \pm 2) \%$ when measured in reflection off the PtMnSb(111) surface. We also show that the SHG polarization turns by about $90^{\circ}$ when changing from a demagnetized to a magnetized state of the Heussler alloy PtMnSb $[17,18]$.

Such big effects are characteristic for materials with strong electronic anisotropies, like some of the Heussler alloys. These are known for their asymmetric electronic structure with regard to the electron spin orientation $[19,20]$. They exhibit half-metallic spin magnetism, which means that for the majority spin direction they behave like metals while they are insulators for the minority spins. Thus at room temperature the conduction electrons should be spin polarized, giving rise to unique magneto-optical properties, as evidenced for example by a large linear Kerr angle of $1.11^{\circ}$ at $633 \mathrm{~nm} \mathrm{[17].} \mathrm{It} \mathrm{was}$ for this reason that we selected PtMnSb for testing the influence of magnetism on SHG. Notice, however, that a PtMnSb crystal has $F 43 m$ symmetry [21]. The lack of inversion symmetry implies that the $\mathrm{SH}$ yield contains both surface and bulk contributions.

The experiments were carried out in air using a straightforward setup similar to the one discussed elsewhere [14,22]. Frequency doubled light of a $Q$-switched Nd:YAG laser (wavelength $532 \mathrm{~nm}$, pulse duration $\approx 6$ $\mathrm{ns}$, intensity at the sample surface $\approx 0.5 \times 10^{6} \mathrm{~W} / \mathrm{cm}^{2}$ ) was reflected at a $75^{\circ}$ angle of incidence off the (111) surface of a PtMnSb single crystal, magnetized to saturation parallel to the plane of incidence. Polarization control of both incident fundamental and reflected $\mathrm{SH}$ light was achieved by means of Glan polarizers and half- or quarter-wave Fresnel rhombs. The SH radiation at 266 $\mathrm{nm}$ was separated from the fundamental using Schott UG-5 filters, and detected by a solar-blind CsTe photomultiplier whose signals were recorded with a gated integrator and then digitally processed.

First we will discuss the results on magnetic circular dichroism. In Fig. 1 an example of the total SHG yield 


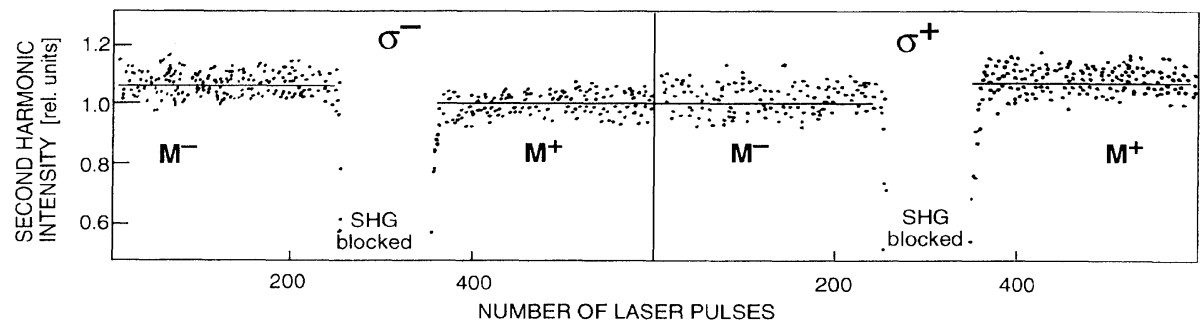

FIG. 1. Raw data showing the magnetic circular dichroism for SHG in reflection off a PtMnSb(111) surface (from Ref. [22]). The $(+)$ and $(-)$ signs indicate parallel or opposite orientation of the axial vectors. The dots represent data accumulated over 50 cycles. Each cycle consists of 250 laser shots for one magnetization, 100 shots with $\mathrm{SH}$ light blocked by a filter, and 250 shots for the opposite magnetization direction.

is shown for right $\left(\sigma^{+}\right)$and left $\left(\sigma^{-}\right)$circularly polarized light defined with regard to the two opposite magnetization directions $(+)$ and $(-)$, respectively. As expected, the largest SHG signal is obtained whenever photon spin and magnetization are oriented parallel, while the yield is smaller for opposite orientation. Defining a dichroitic asymmetry

$$
\Delta=2\left(I^{-}-I^{+}\right) /\left(I^{-}+I^{+}\right),
$$

where the superscript + or - refers to either circular polarization or magnetization direction, the measurements yield $\Delta=(7 \pm 1) \%$.

This effect can be qualitatively understood from the band structure of PtMnSb [20]. Even though no real transitions take place in SHG, the transition probabilities to the manifold of intermediate states, and therefore the respective spin-orbit selection rules, determine the efficiency of the process [12]. We suggest that an unequal population of the ground state in combination with resonance enhancement causes the imbalanced response to the left and right circularly polarized light. According to the discussion in Ref. [20], the uppermost state of the valence band $\left(\Gamma_{4}\right)$ for the minority spin direction is split under spin-orbit interaction with the result that the $m=+1$ orbital component lies above the Fermi level. In contrast, for majority spins the occupation is equally distributed over all magnetic sublevels. Thus for the minority band, $\sigma^{+}$excitation is more important than $\sigma^{-}$excitation. In addition, the $\Gamma_{1}(m=0)$ level may act along the $\Gamma-L$ direction as a nearly resonant first intermediate state. As a result, the hyperpolarizability generated by the right circular fundamental must be stronger than that for the opposite polarization.

The asymmetric total SHG yield for left and right circularly polarized fundamental light implies a change of the ellipticity of the emitted $\mathrm{SH}$ radiation. This is demonstrated in Fig. 2 where the $\mathrm{SH}$ is polarization analyzed with regard to the plane of incidence for $\sigma^{+}$and $\sigma^{-}$fundamental radiation. The experimental data are well described by the solid and dashed lines which were calculated by convoluting the $\mathrm{SH}$ polarization ellipses and the cosine squared transmission of the analyzing
Glan prism. The magnetic influence shows up in two effects: (1) The area under the $\sigma^{+}$curve is larger than that under the $\sigma^{-}$curve. This corresponds to the change of the total yield shown in Fig. 1. (2) The SH polarization ellipses are tilted towards each other by about $13^{\circ}$ [23], which is the NOLIMOKE analog to the MOKE rotation for linearly polarized light. Although the limited accuracy of the data in Fig. 2 precludes a more quantitative interpretation of the rotation, the difference in SHG yield and ellipticity between $\sigma^{+}$and $\sigma^{-}$excitation indicates the resonance enhancement for some $\chi^{(2)}$ tensor elements, as suggested above.

Next, we present data for linearly polarized incident light. The most dramatic effect is obtained when comparing the results for a demagnetized sample, shown in Fig. 3(a), to those with the sample magnetized to saturation in Fig. 3(b). In the demagnetized case, the major axis of the $\mathrm{SH}$ polarization ellipse is parallel to the $p$ polarization of the fundamental, as seen in Fig. 3(a). This corresponds to an asymmetric electron oscillation perpendicular to the surface plane, resulting in a large SHG yield $[11,24]$. The motion in the surface plane is much less restricted by symmetry, thus minimizing its contributions to the nonlinear polarizability. Hence we observe a

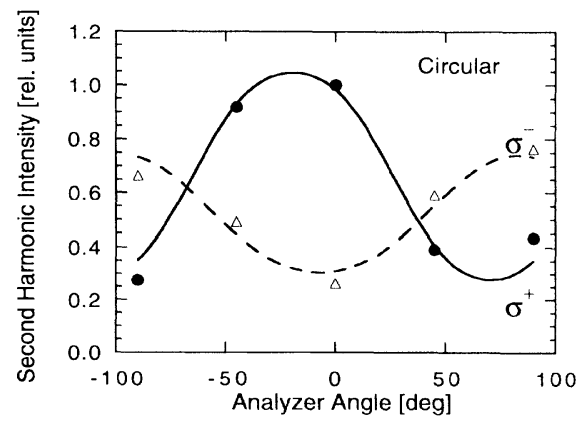

FIG. 2. Polarization analysis of SHG with circularly polarized incident light. The solid and dashed lines are fits to the data with $\sigma^{+}$and $\sigma^{-}$excitation, obtained by a convolution of the respective SH ellipse and the analyzer transmission lellipticity: $\epsilon^{2}\left(\sigma^{+}\right)=0.987$ and $\epsilon^{2}\left(\sigma^{-}\right)=0.940$ l. 


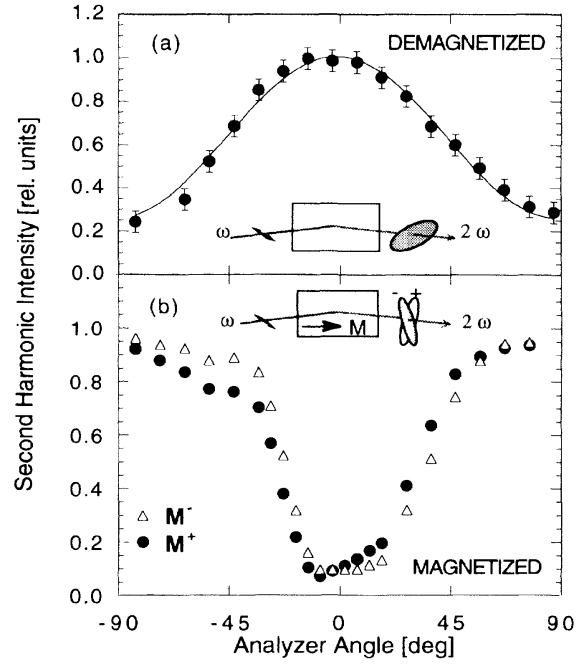

FIG. 3. Polarization analysis of SHG with p-polarized fundamental light. The analyzer angle is defined with regard to the plane of incidence. (a) Demagnetized sample; the solid line is a convolution of the $\mathrm{SH}$ ellipse and the analyzer transmission (ellipticity: $\epsilon^{2}=0.981$ ). (b) Data from fully magnetized sample for opposite magnetization directions. The respective orientations of the $\mathrm{SH}$ polarization ellipse in the demagnetized and magnetized cases are illustrated in the two insets.

minimum of the $\mathrm{SH}$ polarization ellipse perpendicular to the incident $p$ polarization, along the surface plane [Fig. $3(\mathrm{a})$ ]. The situation changes completely when the sample is magnetized to saturation, as can be seen from Fig. 3(b). Now, the major axis of the $\mathrm{SH}$ polarization is perpendicular to the $p$-polarized fundamental, while there is very little SHG yield normal to the surface. This turn of the $\mathrm{SH}$ polarization ellipse by $90^{\circ}$ between the demagnetized and magnetized state is illustrated in the insets of Figs. 3(a) and 3(b), and proves the symmetry-breaking effect of the magnetization [11,16]. In a classical picture, the magnetization restricts the oscillation of the electrons perpendicular to the surface and induces an anisotropic motion in the surface plane. In the SHG formalism, which is beyond the scope of this Letter, the transition from $3 m$ symmetry in the demagnetized to $m$ symmetry in the magnetized state will lead to different tensor components entering the nonlinear susceptibility $\chi^{(2)}[11$, 12,22]. A complication may arise from magnetostriction which we cannot rule out at present, and which seems to be unknown.

Superimposed to the turn of the $\mathrm{SH}$ polarization ellipse when magnetizing the sample is the effect of the two opposite magnetization directions. As can be seen from the data and the inset in Fig. 3(b), there is an additional rotation of the $\mathrm{SH}$ polarization ellipse, depending on whether the magnetization directions $M^{+}$and $M^{-}$are parallel or antiparallel to the $k$ vector of the light. Instead of relying on the difference of a fit to the data for $M^{+}$and

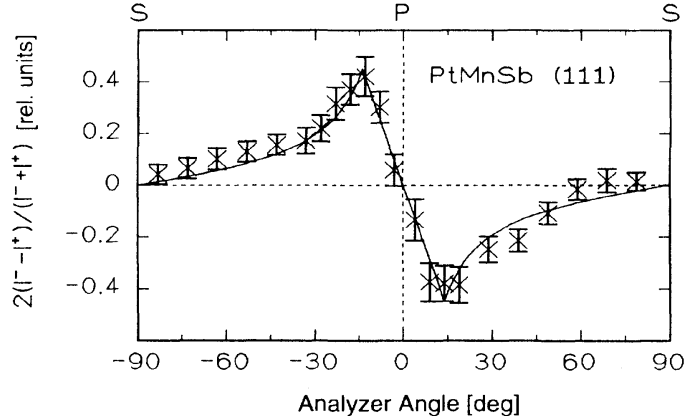

FIG. 4. Dependence of the asymmetry parameter $\Delta$ on the SH polarization angle ( $p$-polarized excitation). The solid line represents a fit to the data as discussed in text (from Ref. [22]).

$M^{-}$in Fig. 3(b), the asymmetry parameter $\Delta$ given by Eq. (1) is used for a phenomenological analysis. When calculating $\Delta$ from the data in Fig. 3(b), the dependence on SH analyzer angle shown in Fig. 4 is obtained. Defining a rotation angle $\alpha$, the variation of $\Delta$ with the analyzer angle $\beta$ follows from geometrical considerations [22]:

$$
\Delta=\left\{\begin{array}{l}
A \tan \alpha / \tan \beta \text { for }-90^{\circ}<\beta<-\alpha, \\
A \tan \beta / \tan \alpha \text { for }-\alpha<\beta<+\alpha, \\
A \tan \alpha / \tan \beta \text { for }+\alpha<\beta<+90^{\circ}
\end{array}\right.
$$

Here, $A$ is a normalization constant, and $\beta$ is the $\mathrm{SH}$ analyzer angle with regard to the plane of incidence. From the fit of these functions to the data in Fig. 4 we obtain a rotation angle of $(28 \pm 4)^{\circ}$ between the $\mathrm{SH}$ polarization ellipses for $M^{+}$and $M^{-}$. We define half of this angle as the nonlinear Kerr angle $\alpha=(14 \pm 2)^{\circ}$. It agrees well with the rotation derived from the data in Fig. 2 for circularly polarized incident light. The qualitative interpretation of this rotation is identical to the one discussed above for circular dichroism. When describing the linear polarization of the fundamental light in terms of two counterrotating circular components, one of which has larger SHG gain, the resultant polarization ellipses of the $\mathrm{SH}$ will be rotated against each other [25]. A quantitative explanation of the size of the nonlinear Kerr angle will have to await theoretical calculations along the lines of Hübner and Bennemann [12] and de Groot and coworkers $[19,20]$.

In summary, we have experimentally demonstrated that magnetic ordering will cause two effects on the SHG yield in reflection: (1) It will alter the symmetry of the nonlinear polarizability. For PtMnSb, this leads to a turn of the polarization ellipse of the $\mathrm{SH}$ light by about $90^{\circ}$. (2) It causes magnetic circular dichroism in SHG and a large nonlinear Kerr angle, in the case of $\mathrm{PtMnSb}$ $(14 \pm 2)^{\circ}$. A comprehensive theory should include both effects and open the possibility to extract the spin-orbit interaction strength from SHG data. Comparison with 
band structure calculations suggests that the large effects observed for $\mathrm{PtMnSb}$ are caused by a combination of different ground state population and resonance enhancement for minority and majority bands. To test this conjecture, SHG investigations must now be carried out on other magnetic materials, among others on $\mathrm{Ni}$, for which theoretical predictions exist [12]. Notwithstanding the detailed mechanism, the results presented in this Letter show the great potential that SHG holds for the field of magnetism. Combined with its high surface sensitivity, its independence on surrounding conditions, and its potential of "viewing" through thin overlayers, this technique is well suited for studying the magnetization of thin films, multilayers, and surfaces.

We gratefully acknowledge the contribution of $\mathrm{J}$. C. Zink to this work. We also thank K.-H. Bennemann and W. Hübner for helpful discussions. This work was supported by the Deutsche Forschungsgemeinscahft, SFB 6, by the National Science Foundation, the Welch Foundation, and the Texas Higher Education Coordinating Board.

*Also with ELIGHT Laser Systems GmbH, Teltow/Berlin, Germany.

[1] E. Hagn and E. Zech, Phys. Rev. B 29, 1148 (1984).

[2] T. R. McGuire and R. I. Potter, IEEE Trans. Magn. 11, 1018 (1975).

[3] B. T. Thole, G. van der Laan, and G. A. Sawatzky, Phys. Rev. Lett. 55, 2086 (1985).

[4] G. Schütz et al., Phys. Rev. Lett. 58, 737 (1987); Z. Phys. B 73, 67 (1988); Phys. Rev. Lett. 62, 2620 (1989).

[5] C. T. Chen, F. Sette, Y. Ma, and S. Modesti, Phys. Rev. B 42, 7262 (1990).

[6] Y. Wu, J. Stöhr, B. D. Hermsmeier, M. G. Samant, and D. Weller, Phys. Rev. Lett. 69, 2307 (1992).

[7] L. Baumgarten, C. M. Schneider, H. Petersen, F. Schäfers, and J. Kirschner, Phys. Rev. Lett. 65, 492 (1990).
[8] G. van der Laan, M. A. Hoyland, M. Surman, C. F. J. Flipse, and B. T. Thole, Phys. Rev. Lett. 69, 3827 (1992).

[9] S. D. Bader and C. Liu, J. Vac. Sci. Technol. A 9, 1924 (1991); E. R. Moog and S. D. Bader, Superlattices Microstruct. 1, 543 (1985).

[10] J. Frey, R. Frey, and C. Flytzanis, Phys. Rev. B 45, 4056 (1992).

[11] Ru-Pin Pan, H. D. Wei, and Y. R. Shen, Phys. Rev. B 39, 1229 (1989).

[12] W. Hübner and K.-H. Bennemann, Phys. Rev. B 40, 5973 (1989); Surf. Sci. 242, 299 (1991); W. Hübner, Phys. Rev. B 42, 11553 (1990).

[13] O. A. Aktsipetrov, O. V. Braginskii, and D. A. Esikov, Kvant. Elektron. 17, 320 (1990) [Sov. J. Quantum Electron. 20, 259 (1990)].

[14] J. Reif, J. C. Zink, C.-M. Schneider, and J. Kirschner, Phys. Rev. Lett. 67, 2878 (1991).

[15] P. Carra, B. T. Thole, M. Altarelli, and X. Wang, Phys. Rev. Lett. 70, 694 (1993).

[16] L. M. Falicov and J. Ruvalds, Phys. Rev. 172, 498 (1968).

[17] P. G. van Engen, K. H. J. Buschow, R. Jongebreur, and M. Erman, Appl. Phys. Lett. 42, 202 (1983).

[18] C. Rau and A. R. Köymen, in Conference on Optoelectronic Materials and Devices, SPIE Proceedings Vol. 836 (SPIE-International Society for Optical Engineering, Bellingham, WA, 1987), p. 99.

[19] R. A. de Groot, Physica (Amsterdam) 172B, 45 (1991); Europhys. News 23, 146 (1992).

[20] J. H. Wijngaard, C. Haas, and R. A. de Groot, Phys. Rev. B 40, 9318 (1989).

[21] K. Watanabe, Trans. Jpn. Inst. Met. 17, 220 (1976); R. A. de Groot, F. M. Mueller, P. G. van Engen, and K. H. J. Buschow, Phys. Rev. Lett. 50, 2024 (1983).

[22] J. C. Zink, dissertation, Freie Universität Berlin, 1992.

[23] The additional $90^{\circ}$ phase shift reflects the opposite helicity of the fundamental when recording the $\mathrm{SH}$ polarization with a linear analyzer.

[24] A. Liebsch, Phys. Rev. Lett. 61, 1233 (1988).

[25] J. L. Erskine and A. E. Stern, Phys. Rev. B 8, 1239 (1973). 